\title{
Anterior gradient 2 downregulation in a subset of pancreatic ductal adenocarcinoma is a prognostic factor indicative of epithelial-mesenchymal transition
}

\author{
Yusuke Mizuuchi ${ }^{1,2}$, Shinichi Aishima ${ }^{1}$, Kenoki Ohuchida ${ }^{3}$, Koji Shindo ${ }^{1,2}$, Minoru Fujino ${ }^{1}$, Masami Hattori ${ }^{1}$, \\ Tetsuyuki Miyazaki ${ }^{1}$, Kazuhiro Mizumoto ${ }^{4}$, Masao Tanaka ${ }^{3}$ and Yoshinao Oda ${ }^{1}$
}

Anterior gradient 2 (AGR2), a member of the protein disulfide isomerase family, has been implicated in various cancers including pancreatic ductal adenocarcinoma (PDAC) and is known to promote cancer progression. However, the prognostic value of AGR2 expression and the interaction with epithelial-mesenchymal transition (EMT) remain unclear. We investigated the clinical significance of AGR2 and EMT markers in PDAC patients by immunohistochemical analyses. Although AGR2 expression was not observed in normal pancreas, all pancreatic precursor neoplastic lesions were positive for AGR2, even at the earliest stages, including pancreatic intraepithelial neoplasia-1A, AGR2 expression was reduced in $27.7 \%$ (54/195 cases) of PDAC patients. AGR2 downregulation correlated with EMT markers (vimentin overexpression and reduced membranous E-cadherin expression), high Union for International Cancer Control stage $(P<0.0001)$, high histological cellular grade $(P<0.0001)$, and adverse outcome $(P<0.0001)$. In vitro, targeted silencing of AGR2 in cancer cells using siRNA reduced cell proliferation, colony formation, cell invasiveness, and migration, but did not alter EMT markers. To confer a more aggressive phenotype and induce EMT in PDAC cells, we co-cultured PDAC cell lines with primary-cultured pancreatic stellate cells (PSCs) and found that AGR2 was downregulated in co-cultured PDAC cells compared with PDAC monocultures. Treatment with transforming growth factor beta-1 (TGF- $\beta$ ), secreted from PSCs, decreased AGR2 expression, whereas inhibition of TGF- $\beta$ signaling using recombinant soluble human TGF- $\beta$ receptor type II and TGF- $\beta$-neutralizing antibodies restored AGR2 expression. We conclude that AGR2 downregulation is a useful prognostic marker, induced by EMT, and that secreted TGF- $\beta$ from PSCs may partially contribute to AGR2 downregulation in PDAC patients. AGR2 downregulation does not induce EMT or a more aggressive phenotype, but is a secondary effect of these processes in advanced PDAC.

Laboratory Investigation (2015) 95, 193-206; doi:10.1038/labinvest.2014.138; published online 24 November 2014

Pancreatic ductal adenocarcinoma (PDAC) is one of the leading causes of cancer death in the United States, with an overall survival rate of 3-5\%. ${ }^{1}$ Early recurrence following resection is associated with high mortality, and to date, few treatment options are available. Thus, the development of novel markers for early detection and prognosis prediction is necessary to improve the management of patients with PDAC. Understanding of the progression from precursors to invasive disease has significantly advanced, following the identification of three important precursors, including pancreatic intraepithelial neoplasia (PanIN), intraductal papillary muci- nous neoplasms (IPMNs), and mucinous cystic neoplasms. However, the mechanisms underlying the progression from these precursors to invasive disease remain unclear. ${ }^{2-4}$

Anterior gradient genes were first identified in Хenopus laevis and are named according to their specific expression patterns during embryonic development. ${ }^{5,6}$ Anterior gradient 2 (AGR2) homolog expression is responsible for the development of the cement gland, a glandular organ in frog embryos, and for limb regeneration in newts. ${ }^{7,8}$ In humans, the AGR2 gene lies at chromosomal position $7 \mathrm{p} 21.3$, and gives rise to an 18 -kDa-sized protein. AGR2 is classified as a member of

\footnotetext{
${ }^{1}$ Department of Anatomic Pathology, Graduate School of Medical Sciences, Kyushu University, Fukuoka, Japan; ${ }^{2}$ Reserch Fellow of Japan Society for the Promotion of Science, Tokyo, Japan; ${ }^{3}$ Department of Surgery and Oncology, Graduate School of Medical Sciences, Kyushu University, Fukuoka, Japan and ${ }^{4}$ Kyushu University Hospital Cancer Center, Fukuoka, Japan

Correspondence: Professor Y Oda, MD, PhD, Department of Anatomic Pathology, Graduate School of Medical Sciences, Kyushu University, 3-1-1 Maidashi, Fukuoka 812-8582, Japan.

E-mail: oda@surgpath.med.kyushu-u.ac.jp

Received 16 February 2014; revised 28 August 2014; accepted 22 September 2014
} 
the protein disulfide isomerase family, involved in protein maturation and folding, on the basis of structural similarity. ${ }^{9}$ Several studies have shown that AGR2 is overexpressed and promoted tumor progression in numerous human malignancies affecting the ovary, breast, lung, prostate, and pancreas. ${ }^{10-14}$

In PDAC, Ramanchandran et al. ${ }^{14}$ reported that AGR2 expression was elevated, even in PanIN1A, the earliest precursor lesion of PDAC. In this context, AGR2 promotes cancer cell proliferation, invasion, and resistance to chemotherapy, suggesting that AGR2 promotes cancer progression; however, the mechanisms underlying AGR2 function in advanced PDAC remain unclear. The prognostic value of AGR2 expression in PDAC is controversial. ${ }^{15}$ Previous reports have also shown that AGR2 promotes dissemination of pancreatic cancer via regulation of lysosomal proteases (cathepsin B and D); however, conversely, AGR2 expression is decreased in PDAC compared with PanIN. ${ }^{16}$

Epithelial-mesenchymal transition (EMT), a well-known hallmark of highly invasive cancer cells, is a critical phenotypic alteration involved in the morphological change from epithelial to mesenchymal phenotype. Emerging evidence suggests that the acquisition of a mesenchymal phenotype has a key role in migration, invasion, resistance to chemoradiotherapy, and extravasation of cancer cells from the primary tumor microenvironment. ${ }^{17}$ Other studies have revealed that decreased membranous expression and cytoplasmic translocation of E-cadherin provide an indication of EMT. ${ }^{18,19}$ Furthermore, EMT is correlated with high histological grade in breast and pancreatic cancer. ${ }^{20}$ To the best of our knowledge, however, there are no studies addressing the correlation between AGR2 expression and EMT induction.

The tumor microenvironment, which is composed of immune cells, fibroblastic cells, blood vessels, and extracellular matrix in cancer stroma, closely interacts with cancer cells invading the stroma. Pancreatic stellate cells (PSCs), having myofibroblastic morphology in activated state, are located in the pancreatic stroma and have an important role in the pancreatic cancer microenvironment. ${ }^{21}$ The interaction between pancreatic cancer cells and PSCs promotes cancer cell proliferation, migration, and invasion via the secretion of soluble factors, such as transforming growth factor beta-1 (TGF- $\beta 1$ ), epidermal growth factor (EGF), and fibroblast growth factor 2 (FGF-2), which are well-characterized EMT-inducible factors. ${ }^{22-26} \mathrm{~A}$ recent report demonstrated that PSCs also promote EMT in pancreatic cancer cells. ${ }^{27}$

In this study, we aim to verify whether AGR2 expression represents a useful prognostic marker in human PDAC, and whether its downregulation is mediated by EMT induction. We identified a correlation between AGR2 expression, EMT marker expression, and clinic-pathological parameters in PDAC. Our study also shows that preserved AGR2 expression is a favorable prognostic factor in PDAC.

\section{MATERIALS AND METHODS}

\section{Patients and Tissue Samples}

Formalin-fixed, paraffin-embedded surgical resection samples (PDAC, $n=195$, PanIN, $n=15$, and IPMN, $n=39$ ) were obtained from patients who were diagnosed and underwent pancreatic resection at our institution between July 1999 and March 2009. Forty variants of pancreatic cancer including adenosquamous carcinoma $(n=4)$, adenocarcinoma with squamous differentiation $(n=6)$, anaplastic carcinoma $(n=3)$, and acinar cell carcinoma $(n=1)$ were excluded from this study. The study protocol was approved by the Ethics Committee of Kyushu University (approval number 24-222, 25-117) and conformed to the Ethical Guidelines for Human Genome/Gene Research enacted by the Japanese Government and the Declaration of Helsinki. For strict privacy protection, identifying information for all patients was removed before analysis.

\section{Immunohistochemical Analysis}

Primary antibodies used for immunohistochemical (IHC) analysis were as follows: AGR2 (rabbit polyclonal, IMG-5279A, 1/100 dilution, IMGENEX, San Diego, CA, USA), vimentin (mouse monoclonal, Clone V9, 1/25 dilution, DAKO Cytomation, Glostrup, Denmark) and E-cadherin (mouse monoclonal, 610181, 1/1000 dilution, BD Biosciences, Franklin Lakes, NJ, USA). Antibodies were diluted in 5\% dry skimmed milk in PBS. In brief, $5-\mu \mathrm{m}$-thick tissue sections were deparaffinized in xylene and dehydrated in ethanol. Endogenous peroxidase activity was blocked by incubation in methanol containing $0.3 \% \mathrm{H}_{2} \mathrm{O}_{2}$ for $30 \mathrm{~min}$. Antigen retrieval was achieved by boiling slides in a microwave in $10 \mathrm{mM}$ citrate buffer $(\mathrm{pH}$ 6.0) for 20 min (AGR2 and E-cadherin only). The slides were then incubated with primary antibodies at $4{ }^{\circ} \mathrm{C}$ overnight and subsequently incubated with biotin-free horseradish peroxidase enzyme-labeled polymer (Envision plus System, DAKO Cytomation) for $40 \mathrm{~min}$ at room temperature. The labeled antigens were visualized using 3,3'-diaminobenzidine tetrahydrochloride as a chromogen. Counterstaining was performed with hematoxylin. Appropriate positive and negative controls were performed for all antibodies. Nonspecific staining was not observed in any negative-control sections.

\section{Evaluation of Immunohistochemical Staining}

All tissue sections were assessed by two investigators (YM and SA) who were blinded to patient clinicopathological results. AGR2 immunoreactivity was determined using an intensity score, graded on a scale from 0 to 3 , and a proportional score, corresponding to the percentage of stained cells $(0$ : no staining, $1:<20 \% ; 2: 20-50 \%$, and $3:>50 \%$ ), as previously described. ${ }^{16}$ The total score was the product of intensity and the proportional score. AGR2 high-expressing patients scored between 4 and $6(n=139)$ and low-expressing patients between 0 and $3(n=54)$. Membranous immunoreactivity for E-cadherin was categorized as high expression $(>50 \%$ of 
cells staining positive, similar to normal epithelial cells) or low expression $(<50 \%$ of cells staining positive), as previously described. ${ }^{28}$ Cytoplasmic expression of vimentin was categorized as positive if any cancer cell exhibited positive immunoreactivity and as negative if there was complete absence of vimentin staining.

\section{Cells and Culture Conditions}

The following pancreatic cancer cell lines were used in this study: HPC-3, H48N (Dr H Iguchi, National Shikoku Cancer Center, Matsuyama, Japan), Panc-1 (Riken Cell Bank, Tsukuba, Japan), SUIT-2, MIA PaCa-2 (Japanese Cancer Resource Bank, Osaka, Japan), HS766T, BxPC-3, Capan-2, Aspc-1, and CFPAC-1 (American Type Culture Collection, Manassas, VA, USA). Human PSCs were isolated from PDAC surgical specimens using the outgrowth method as previously described. ${ }^{21}$ Primary cultures of PSCs derived from PDAC patients were established in our laboratory. These cells exhibited a fibroblast-like morphology, and immunofluorescence analysis revealed that the majority of cells were positive for vimentin and alpha-smooth muscle actin (>90\%), markers of activated fibroblasts and glial fibrillary acidic protein, a marker of PSCs, and negative for cytokeratin 19, a marker of epithelial cells (Supplementary Figure 1). All established PSCs were used between passages 3 and 7. All cells were maintained in Dulbecco's modified Eagle's medium (DMEM) (Life Technology, Grand Island, NY, USA) supplemented with $10 \%$ fetal bovine serum (FBS) (Invitrogen, Carlsbad, CA, USA), streptomycin $(100 \mathrm{mg} / \mathrm{ml})$ and penicillin $(100 \mathrm{mg} / \mathrm{ml})$ and grown at $37^{\circ} \mathrm{C}$ in a humidified atmosphere containing $10 \% \mathrm{CO}_{2}$.

\section{Western Blotting}

Protein was extracted from cell lines using lysis buffer (PROPREP Protein Extraction Solution, iNtRON Biotechnology, Seongnam, South Korea), and lysates $(20 \mu \mathrm{g})$ were separated by SDS-PAGE and transferred to polyvinylidene difluoride membranes (Immobilon P, Millipore, Bedford, MA, USA). Membranes were blocked with 5\% dry skimmed milk and incubated with primary antibodies; anti-AGR2 rabbit polyclonal antibody (1:500, IMGENEX), anti-E-cadherin mouse polyclonal antibody (1:5000, BD Biosciences), anti-vimentin mouse monoclonal antibody (1:500, DAKO Cytomation), anti-phospho-Smad2 (Ser465/467)/Smad3 (Ser423/425) rabbit monoclonal antibody (\#8828, 1:1000, Cell Signaling Technology, Danvers, MA, USA), anti-Smad2/3 rabbit monoclonal antibody (\#8685, 1:1000, Cell Signaling Technology), antiphospho-p44/42 MAPK (Erk1/2) (Thr202/Tyr204) rabbit monoclonal antibody (\#4370, 1:2000, Cell Signaling Technology), and anti-p44/42 MAPK (Erk1/2) rabbit monoclonal antibody (\#4695, 1:1000, Cell Signaling Technology) using the SNAP i.d. Protein Detection System (Millipore) in accordance with the manufacturer's instructions. Anti-glyceraldehyde 3-phosphate dehydrogenase antibody (mouse monoclonal, sc32233, 1/5000 dilution, Santa Cruz Biotechnology,
Santa Cruz, CA, USA) was used as an internal control. Membranes were then incubated with anti-rabbit IgG (\#7074, 1/5000 dilution, Cell Signaling Technology), anti-mouse IgG1 (sc-2061, 1/3000 dilution, Santa Cruz Biotechnology), and anti-mouse IgG2a (sc-2061, 1/3000 dilution, Santa Cruz Biotechnology). Immunoreactive signals were detected using ECL Prime (GE Healthcare, Buckinghamshire, UK) and images were acquired using a ChemiDoc XRS (Bio-Rad Laboratories, Hercules, CA, USA).

\section{Quantitative RT-PCR (qRT-PCR)}

qRT-PCR was performed as previously described. ${ }^{29}$ Total RNA was isolated from cancer cell lines using the High Pure RNA Isolation Kit (Roche Diagnostics, Mannheim, Germany) with DNase I (Roche Diagnostics) treatment, in accordance with the manufacturer's instructions. RNA was quantified by measuring absorbance at $260 \mathrm{~nm}$, and purity was evaluated from the 260/280 ratio of absorbance using a NanoDrop ND-1000 spectrophotometer (NanoDrop Technologies, Wilmington, DE, USA). qRT-PCR was performed using the QuantiTect SYBR Green Reverse Transcription-PCR kit (Qiagen, Tokyo, Japan) and a CFX96 Touch Real-Time PCR Detection System (Bio-Rad Laboratories). Data are presented as relative expression normalized to $18 \mathrm{~S}$ rRNA levels. The primer sequences are listed in Supplementary Table 1.

\section{Immunofluorescence Staining}

Primary-cultured PSCs were seeded in 35-mm glass-bottom dishes (Matsunami, Osaka, Japan) $\left(2 \times 10^{4}\right.$ cells/dish $)$ in DMEM with $10 \%$ FBS and incubated for $24 \mathrm{~h}$. Cells were then fixed with methanol, blocked with 3\% bovine serum albumin in PBS, and incubated with either anti-alphasmooth muscle actin antibody (rabbit polyclonal, ab5694, 1/100 dilution, Abcam, Cambridge, MA, USA), antivimentin antibody (mouse monoclonal, clone V9, 1/50 dilution, Dako), anti-glial fibrillary acidic protein antibody (mouse monoclonal, ab10062, 1/100 dilution, Abcam), and anti-cytokeratin 19 antibody (goat polyclonal, sc-33111, 1/5000 dilution, Santa Cruz Biotechnology) overnight at $4{ }^{\circ} \mathrm{C}$. Cells were then incubated with Alexa 488-conjugated anti-mouse IgG (A11029, Molecular Probes, Eugene, OR, USA), 546-conjugated anti-rabbit IgG (A11035, Molecular Probes), or 546-conjugated anti-goat IgG (A11056, Molecular Probes) for $1 \mathrm{~h}$. Nuclear DNA was counterstained with $4^{\prime}, 6$-diamidino-2-phenylindole $(0.05 \mu \mathrm{g} / \mathrm{ml})$ (Dojindo, Kumamoto, Japan). Fluorescence was visualized using an All-in-one Type Fluorescence Microscope (BZ-9000; Keyence Corporation, Osaka, Japan), and images were acquired using BZ-II image analysis software (Keyence).

\section{AGR2 Knockdown by Small-Interfering RNA (siRNA)}

siRNA targeting AGR2 (On-Target plus Smart Pool siRNAssiAGR2) and non-targeting siRNA control (ON-TARGET plus non-targeting siRNA) were purchased from Dharmacon 
(Chicago, IL, USA). Transfection was performed according to the manufacturer's reverse-transfection protocol using Lipofectamine RNAiMAX (Life Technology). In brief, siRNAs and Lipofectamine $(5 \mu \mathrm{l})$ were diluted in $500 \mu \mathrm{l}$ Opti-MEM (Life Technology) without serum, and incubated for $10 \mathrm{~min}$ at room temperature. Cancer cells $\left(3 \times 10^{5}\right)$ were resuspended in $2.5 \mathrm{ml}$ of DMEM supplemented with $10 \%$ FBS without antibiotics. The siRNA and Lipofectamine mixture was added to the diluted cells $(3 \mathrm{ml}$ final volume, final siRNA concentration $2 \mathrm{nM}$ for Aspc-1 cells and $10 \mathrm{nM}$ for Panc-1, Hs766T, Capan-2, and BxPC-3 cells) and seeded in six-well plates $\left(3 \times 10^{5}\right.$ cells/well). After 24 -h incubation, plates were washed and cells were incubated in complete growth medium (DMEM with 10\% FBS and antibiotics) for various time points. Cancer cells were used in subsequent experiments $48 \mathrm{~h}$ post transfection.

\section{Proliferation Assays}

Cancer cell lines were seeded in 24-well plates $\left(1 \times 10^{4}\right.$ cells/ well) (Becton Dickinson, Bedford, MA, USA) in DMEM containing 10\% FBS. Following incubation for the indicated times $(0,1,3$, or 5 days $)$, propidium iodide $(30 \mu \mathrm{mol} / \mathrm{l})$ and digitonin $(600 \mu \mathrm{mol} / \mathrm{l})$ were added to each well to label cell nuclei. Cell proliferation was evaluated by measuring the fluorescence intensity of propidium iodide, as previously described $^{30}$ using an infinite F200 (Tecan, Männedorf, Switzerland).

\section{Colony Formation Assays}

Anchorage-independent growth was assessed by colony formation in soft agar as previously described. ${ }^{31}$ Single-cancer cells $\left(1 \times 10^{4}\right.$ cells $)$ were diluted in DMEM with $10 \%$ FBS and 0.35\% Bacto-Agar (Difco, Detroit, MI, USA), and seeded in six-well plates on top of a $0.7 \%$ agar bottom layer without cells. Cells were incubated for 14 days, and growth medium (DMEM with 10\% FBS) was replaced biweekly. Colonies were stained with crystal violet $(0.005 \%)$ for $20 \mathrm{~min}$ and counted under a light microscope.

\section{Matrigel Invasion and Migration Assays}

Cancer cell migration and invasiveness were assessed by determining the number of cells invading $8-\mu \mathrm{m}$ pore Transwell chambers (BD Biosciences). Transwell inserts were coated with Matrigel (20 $\mu \mathrm{g} /$ well; BD Biosciences, Bedford, MA, USA) for invasion assays, and without coating for migration assays. For indirect co-culture experiments, PDAC cell lines $\left(4 \times 10^{4}\right.$ cells $)$ were resuspended in $250 \mu \mathrm{l}$ of DMEM containing 1\% FBS, and seeded in the upper chamber, which was then placed in a 24-well culture dish containing PSCs $\left(4 \times 10^{4}\right.$ cells) resuspended in $750 \mu \mathrm{l}$ of the same medium. For monoculture experiments using AGR2 knockdown cells, cancer cells $\left(4 \times 10^{4}\right.$ cells $)$ were resuspended in $250 \mu \mathrm{l}$ of DMEM containing $10 \%$ FBS and seeded in the upper chamber, which was placed in a 24-well culture dish containing $750 \mu \mathrm{l}$ of the same medium. Invasion and migrations assays were performed using Aspc-1 and Hs766T cells for 36 and $24 \mathrm{~h}$, respectively. Invading or migrating cancer cells were fixed to the lower surface of the transwell membrane with $70 \%$ ethanol, stained with hematoxylin-eosin and counted in five random fields at $\times 100$ magnification, using an All-in-one Type Fluorescence Microscope (BZ-9000; Keyence) and BZ-II image analysis software (Keyence). Results are expressed as the mean number of cancer cells translocating to the lower surface.

\section{Indirect Co-Culture of Cancer Cells with PSCs}

Indirect co-culture experiments were performed as previously described. ${ }^{27}$ Pancreatic cancer cells $\left(1 \times 10^{5}\right.$ cells $)$ were seeded in six-well plates (BD Biosciences) in $2 \mathrm{ml}$ DMEM supplemented with $10 \%$ FBS. Human primary-cultured PSCs $\left(3 \times 10^{5}\right.$ cells $)$ were seeded into $3-\mu \mathrm{m}$ pore Transwell chambers in $1 \mathrm{ml}$ DMEM with $10 \%$ FBS. The following day, Transwell chambers seeded with PSCs were placed into six-well plates containing pancreatic cancer cells, and incubated up to $48 \mathrm{~h}$ in DMEM with $1 \%$ FBS.

\section{Treatment with Recombinant Proteins}

To identify the factors involved in AGR2 downregulation, we cultured cancer cells in the presence of recombinant human TGF- $\beta 1$, EGF, and FGF-2, which have previously been shown to induce EMT. ${ }^{24-26}$ Cancer cells were seeded in $90-\mathrm{mm}$ dishes $\left(1 \times 10^{5}\right.$ cells/dish $)$ and incubated for $48 \mathrm{~h}$ in DMEM supplemented with $1 \%$ FBS with or without various growth factors $(0.5,1,2$, and $5 \mathrm{ng} / \mathrm{ml}$ recombinant human TGF- $\beta 1$ (R\&D Systems, Oxon, UK), 1,10 , and $100 \mathrm{ng} / \mathrm{ml}$ recombinant human FGF-2 (Sigma, Basel, Switzerland), and $1,10,20$, and $100 \mathrm{ng} / \mathrm{ml}$ recombinant human EGF (Sigma).

\section{Enzyme-Linked Immunosorbent Assay (ELISA) for TGF- $\beta 1$ Levels}

Secreted TGF- $\beta 1$ levels were assayed in cell culture supernatants using the Quantikine human TGF- $\beta 1$ kit (R\&D Systems). Aspc- 1 cells $\left(1 \times 10^{5}\right.$ cells $)$ were plated in six-well plates and co-cultured with or without primary-cultured PSCs $\left(3 \times 10^{5}\right.$ cells $)$ for $24 \mathrm{~h}$ in serum-free DMEM. Supernatants were then collected and analyzed in accordance with the manufacturer's instructions. Cancer cells were co-cultured with PSCs as described above. Each sample was assayed in triplicate and quantification was obtained using a plate reader at $450 \mathrm{~nm}$ (corrected by subtracting absorbance at $540 \mathrm{~nm})$.

\section{Inhibition of TGF- $\beta$ Signaling Using Neutralizing Antibody}

For inhibition of TGF- $\beta$ signaling, PDAC cells were co-cultured with PSCs as described above, and treated with recombinant human TGF- $\beta$ sRII/Fc (rhT $\beta$ sR-II) $(100 \mathrm{ng} / \mathrm{ml}, 341-\mathrm{BR}, \mathrm{R} \& \mathrm{D}$ Systems $){ }^{32}$ TGF- $\beta$ neutralizing antibody $(10 \mu \mathrm{g} / \mathrm{ml}$, MAB1835, R\&D Systems) or mouse control IgG1 (10 $\mu \mathrm{g} / \mathrm{ml}$, MAB002, 

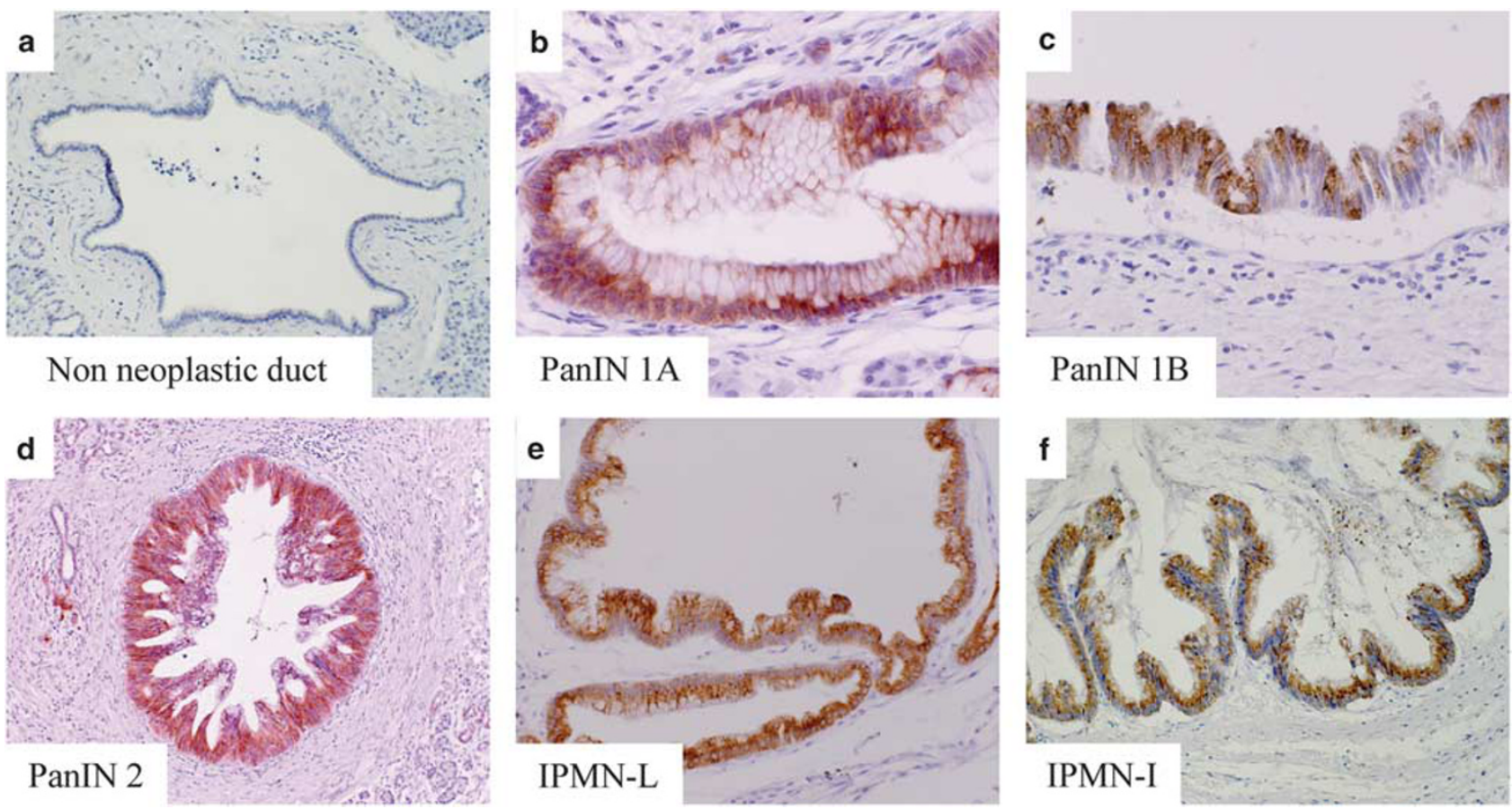

Figure 1 Immunohistochemical analysis of anterior gradient 2 (AGR2) in chronic pancreatitis and pre-cancerous lesions. AGR2 expression was not detected in non-neoplastic ducts of chronic pancreatitis patients (a). AGR2 upregulation was observed in benign PDAC precursor lesions including PanIN1A (b) and IPMN with low-grade dysplasia (e), and expression was retained in more severe precursor lesions (c, $\mathbf{d}$ and $\mathbf{f})$. PanIN, pancreatic intraepithelial neoplasia; IPMN-L, intraductal papillary mucinous neoplasm with low-grade dysplasia; IPMN-I, intraductal papillary mucinous neoplasm with intermediate-grade dysplasia; (original magnification: (a) $\times 100$, (b) $\times 200,(\mathbf{c}) \times 200,(\mathbf{d}) \times 40,(\mathbf{e}) \times 100,(\mathbf{f}) \times 100)$.

R\&D Systems) for $48 \mathrm{~h}$ in DMEM supplemented with $1 \%$ FBS.

\section{Statistical Analysis}

The correlation between two variables was analyzed using the $\chi^{2}$-test or the Fisher exact test, where appropriate. For survival analysis, disease-free survival was adopted as primary end points. The survival correlation was presented using Kaplan-Meier analysis, and the curves were compared using the log-rank test. Linear regression analysis was used to examine the association between mRNA levels of AGR2 and E-cadherin in PDAC cell lines. For in vitro experiments, continuous variables were expressed as the mean \pm s.d. Each experiment was performed in biological triplicate. Comparison between two groups was analyzed using the Student's $t$-test. Statistical significance was defined as $P<0.05$. All statistical analyses were performed using JMP 9.01 software (SAS Institute, Cary, NC, USA).

\section{RESULTS}

AGR2 Expression in PDAC and Pre-Cancerous Lesions

IHC analyses revealed AGR2 staining in the cytoplasm of neoplastic cells. All PDAC precursors were positive for AGR2 expression, even in PanIN1A $(100 \%, 10 / 10)$ and IPMN with low-grade dysplasia $(100 \%, 20 / 20)$, which represent the most benign types of PDAC precursor lesions. AGR2 expression was retained in PanIN2 (100\% 5/5) and IPMN with intermediate-grade dysplasia $(100 \%, 19 / 19)$. In contrast, AGR2 immunoreactivity was not detected in normal pancreatic duct specimens of chronic pancreatitis patients (Figure 1). Clinicopathological findings and IHC characteristics for AGR2 are summarized in Table 1. Of the 195 PDAC patients, AGR2 expression was decreased in 54 patients (27.7\%). Decreased AGR2 expression was associated with high histological grade (G3 (poorly differentiated); refer to structure, growth pattern of the cancer cells, and nuclear atypia) $(P<0.0001)$ and high Union for International Cancer Control stage ( $>$ stage II) $(P=0.0061)$.

\section{Reduced Expression of AGR2 Correlates with Markers of EMT in PDAC}

We observed decreased expression of AGR2 in areas of high histological grade and decreased cell adhesiveness in the tissue of PDAC patient (Figures 2a-h). Therefore, we hypothesized that EMT may be associated with AGR2 downregulation. To assess this hypothesis, we investigated the expression of EMT markers (E-cadherin and vimentin) in serial sections of PDACs by IHC staining. In Table 1 , membranous E-cadherin expression was reduced $(<50 \%)$ in 68 patients $(34.9 \%)$ and cytoplasmic vimentin expression was observed in 36 patients (18.5\%), and AGR2 downregulation 
Table 1 Clinicopathological characteristics of PDAC patients $(n=195)(* P<0.01)$

\begin{tabular}{|c|c|c|c|c|}
\hline \multirow[b]{2}{*}{ Variable } & \multicolumn{2}{|c|}{ AGR2 expression } & \multirow{2}{*}{$\begin{array}{l}\text { Total } \\
(n=195)\end{array}$} & \multirow[b]{2}{*}{$P$-value } \\
\hline & High $(n=141)$ & Low $(n=54)$ & & \\
\hline Age (in years) & $66.5(36-86)$ & $63.7(36-80)$ & & \\
\hline Male/female & $84 / 57$ & $37 / 17$ & 121 & 0.2494 \\
\hline \multicolumn{5}{|l|}{ Location } \\
\hline Head/body and tail & $96 / 43$ & $33 / 21$ & $129 / 63$ & 0.3885 \\
\hline \multicolumn{5}{|l|}{ E-cadherin } \\
\hline High expression & 114 & 13 & 127 & $<0.0001^{*}$ \\
\hline Low expression & 27 & 41 & 68 & \\
\hline \multicolumn{5}{|l|}{ Vimentin } \\
\hline Positive & 14 & 22 & 36 & $<0.0001^{*}$ \\
\hline Negative & 127 & 32 & 159 & \\
\hline Histological grade & & & & $<0.0001^{*}$ \\
\hline G1 (well) & 42 & 2 & & \\
\hline G2 (moderately) & 60 & 9 & & \\
\hline G3 (poorly) & 27 & 38 & & \\
\hline Local invasion & 118/139 (84.9\%) & 49/53 (92.3\%) & & 0.164 \\
\hline Vessel invasion & 111/141 (78.7\%) & $46 / 53(86.8 \%)$ & & 0.2024 \\
\hline Lymph node & 95/141 (67.4\%) & $43 / 54$ (79.6\%) & & 0.0923 \\
\hline \multicolumn{5}{|l|}{ metastasis } \\
\hline \multicolumn{5}{|l|}{ UICC T category } \\
\hline $1 / 2$ & 14 & 5 & 19 & 0.8878 \\
\hline $3 / 4$ & 127 & 49 & 176 & \\
\hline \multicolumn{5}{|l|}{ UICC stage } \\
\hline Stage $|/| I$ & 137 & 47 & 184 & $0.0061^{*}$ \\
\hline Stage III/IV & 4 & 7 & 11 & \\
\hline
\end{tabular}

Abbreviations: AGR2, anterior gradient 2; PDAC, pancreatic ductal adenocarcinoma; UICC, Union for International Cancer Control.

was associated with reduced membranous E-cadherin expression $(P<0.0001)$ and cytoplasmic vimentin expression $(P<0.0001)$. Areas of reduced AGR2 expression were consistent with those exhibiting induction of EMT (decreased membranous expression of E-cadherin and positive cytoplasmic vimentin expression) (Figures $2 \mathrm{c}, \mathrm{d}, \mathrm{g}, \mathrm{h}, \mathrm{k}, \mathrm{l}, \mathrm{o}$ and $\mathrm{p}$ ). These data demonstrate that AGR2 downregulation is correlated with induction of EMT, probing a deeper analysis of the interaction between these two parameters.

\section{Reduced Expression of AGR2 Correlates with Poor Prognosis in PDAC Patients}

We next examined the relationship between AGR2 expression and survival of PDAC patients. Univariate analysis revealed that decreased AGR2 expression was a statistically significant indicator of adverse prognosis for disease-free survival (Figure 3a). Conversely, multivariate analysis revealed that AGR2 downregulation was not an independent predictor of shorter overall survival (Supplementary Table 2). To examine the impact of AGR2 and E-cadherin status on disease-free survival, we determined the disease-free survival rate with combined AGR2 and E-cadherin status (Figures 3b-d). Low AGR2 expression was correlated with poor disease-free survival. Further evaluation on the basis of E-cadherin expression revealed that lower AGR2 expression correlated with a lower survival rate in both high- and low-E-cadherin expression cohorts, although this difference was not statistically significant (Figures $3 \mathrm{c}$ and d). These data suggest that AGR2 downregulation could contribute to poor prognosis, but is partially affected by decreased membranous E-cadherin expression.

\section{AGR2 Expression in Pancreatic Cancer Cell Lines}

Analysis of AGR2 expression in a panel of nine pancreatic cancer cell lines by qRT-PCR revealed significant variation in expression levels. Epithelial-type cancer cell line (high E-cadherin, low vimentin; HS766T, H48N, and BxPC-3) exhibiting cellular adhesiveness retained AGR2 expression, whereas spindle-shaped mesenchymal-type cancer cell line (low E-cadherin, high vimentin; MiaPaCa2, SUIT-2, and HPC-3) expressed relatively low levels of AGR2 (Figure 4a). Linear regression analysis revealed a strong positive correlation between AGR2 and E-cadherin expression at the mRNA level (Figure $4 \mathrm{~b} ; R^{2}=0.7262, P=0.0035$ ). Taken together, these results strengthen the hypothesis that AGR2 downregulation is correlated with EMT induction.

\section{AGR2 Knockdown Using siRNA}

To analyze the function of AGR2 in vitro, we performed targeted knockdown of AGR2 in PDAC cells using anti-AGR2 siRNA. Efficient knockdown of AGR2 transcript ( $>70 \%$ until $120 \mathrm{~h}$ post transfection) was confirmed by qRT-PCR (Figures $5 \mathrm{a}$ and $\mathrm{g}$ ), and reduced AGR2 protein levels were confirmed by western blot $72 \mathrm{~h}$ post transfection (Figure $5 \mathrm{~b}$ ). Targeted inhibition of AGR2 in both Aspc-1 and Hs766T cells led to a significant decrease in anchorage-dependent cell proliferation (Figure 5c), colony formation (Figure 5d), cell migration (Figure 5e), and cell invasiveness (Figure 5f) compared with cells transfected with non-coding negativecontrol RNA $(P<0.05)$, except for the colony formation assay in Hs766T $(P=0.0686)$. AGR2 knockdown did not alter the expression of epithelial or mesenchymal markers (E-cadherin and vimentin, respectively), or transcription factors involved in EMT induction (Snail-1, Snail-2, and ZEB-1) in PDAC cells (Figure 5g), implying that AGR2 does 


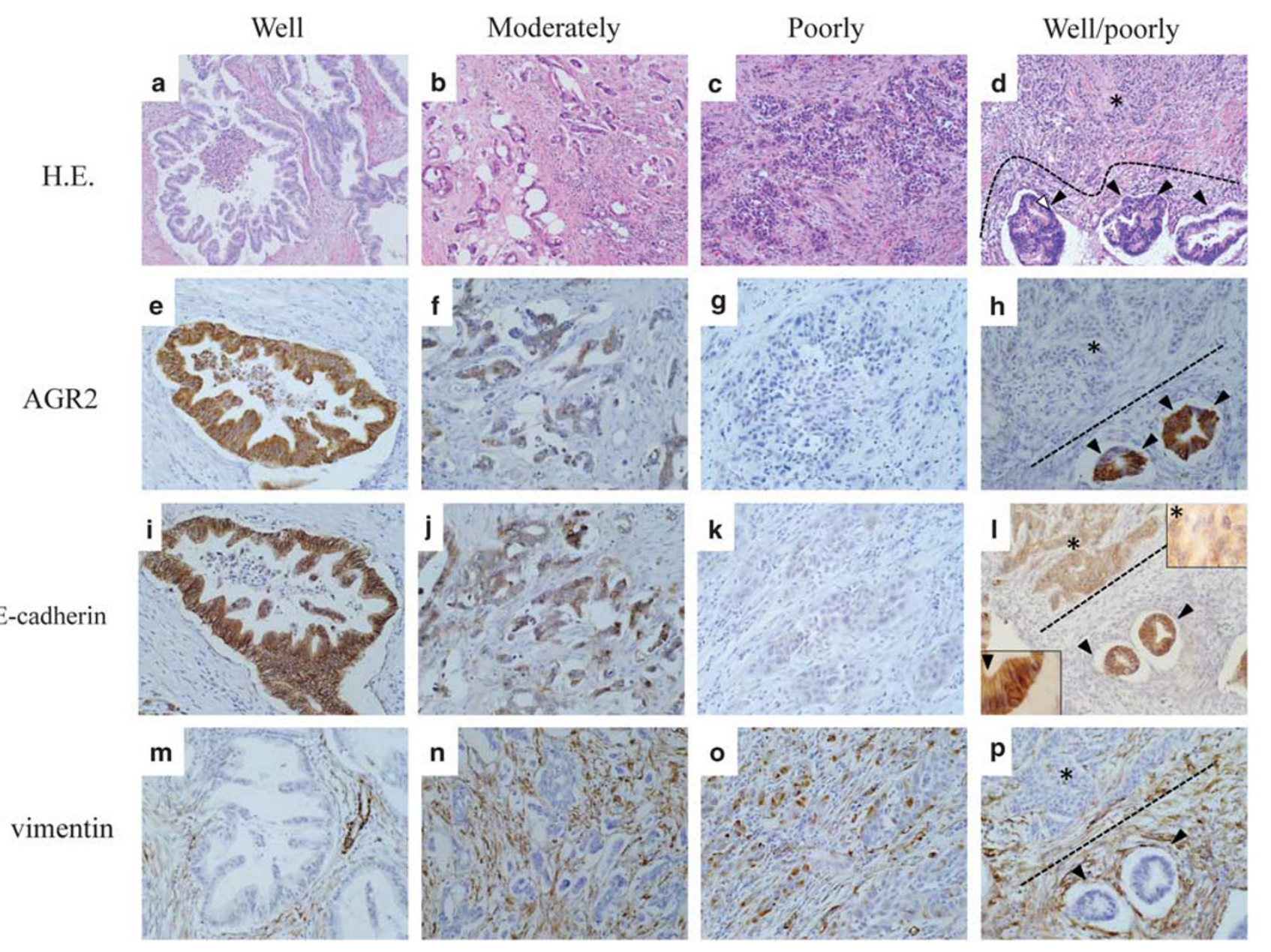

Figure 2 Histological analyses of human PDAC specimens. Representative images of hematoxylin and eosin (H\&E) staining (a-d) and immunohistochemical staining for anterior gradient 2 (AGR2) (e-h), E-cadherin (i-l), and vimentin ( $\mathbf{m}-\mathbf{p})$. Each column of figures represent serial sections of one PDAC patient (a, e, i and $\mathbf{~ m}$ : well differentiated; $\mathbf{b}, \mathbf{f}, \mathbf{j}$ and $\mathbf{n}$ : moderately differentiated; $\mathbf{c}, \mathbf{g}$, $\mathbf{k}$ and $\mathbf{o}$ : poorly differentiated; $\mathbf{d}$, $\mathbf{h}, \mathbf{I}$ and p: admixed components of well- (arrowhead) and poorly (asterisk) differentiated adenocarcinoma. Low-histological-cellular-grade (well or moderately differentiated) PDAC retained AGR2 and membranous E-cadherin expression, whereas vimentin expression was absent. Cytoplasmic AGR2 expression and membranous E-cadherin expression were decreased in parallel with cancer de-differentiation, and positive cytoplasmic vimentin expression was observed in high-histological-cellular-grade (poorly differentiated) PDAC. E-cadherin expression is seen at the membrane and in the cytoplasm of the well-differentiated carcinoma, but is decreased in the membrane of the poorly differentiated carcinoma (I; insets). Original magnification: H\&E staining $\times 100$, IHC $\times 200$

not directly regulate EMT induction in PDAC cells. These results are consistent with previous reports, but not our IHC data. It was suggested that correlation with adverse prognosis and EMT induction in low-AGR2-expressing PDAC patients by our IHC analysis was not due to loss of AGR2 function itself. On the basis of these observations, we hypothesize that other molecules involved in PDAC progression function as upstream negative regulators of AGR2.

\section{AGR2 Expression Decreased in PDAC Cell Lines Co-Cultured with PSCs}

Among the mechanisms promoting cancer progression, cancer-stromal interactions have a crucial role in cancer cells invading the tumor microenvironment, but not in intraepithelial lesions. To investigate the relationship between AGR2 downregulation, tumor microenvironment and EMT induction, we performed co-culture experiments, in which PDAC cells were indirectly co-cultured with human primarycultured PSCs. Co-culture of PDAC cells with primary PSCs induced EMT, as evidenced by a decrease in E-cadherin and increase in vimentin, Snail-1, Snail-2, and ZEB-1 mRNA levels, compared with cancer cell monocultures (Figures 6b and c), and E-cadherin and vimentin expression was slightly altered in protein levels. AGR2 expression was decreased in co-cultured PDAC cells at the mRNA and protein levels (Figures $6 \mathrm{a}$ and $\mathrm{d}$ ). The results of these indirect co-culture experiments suggest that soluble factors secreted from PSCs contribute to AGR2 downregulation in cancer cells. 

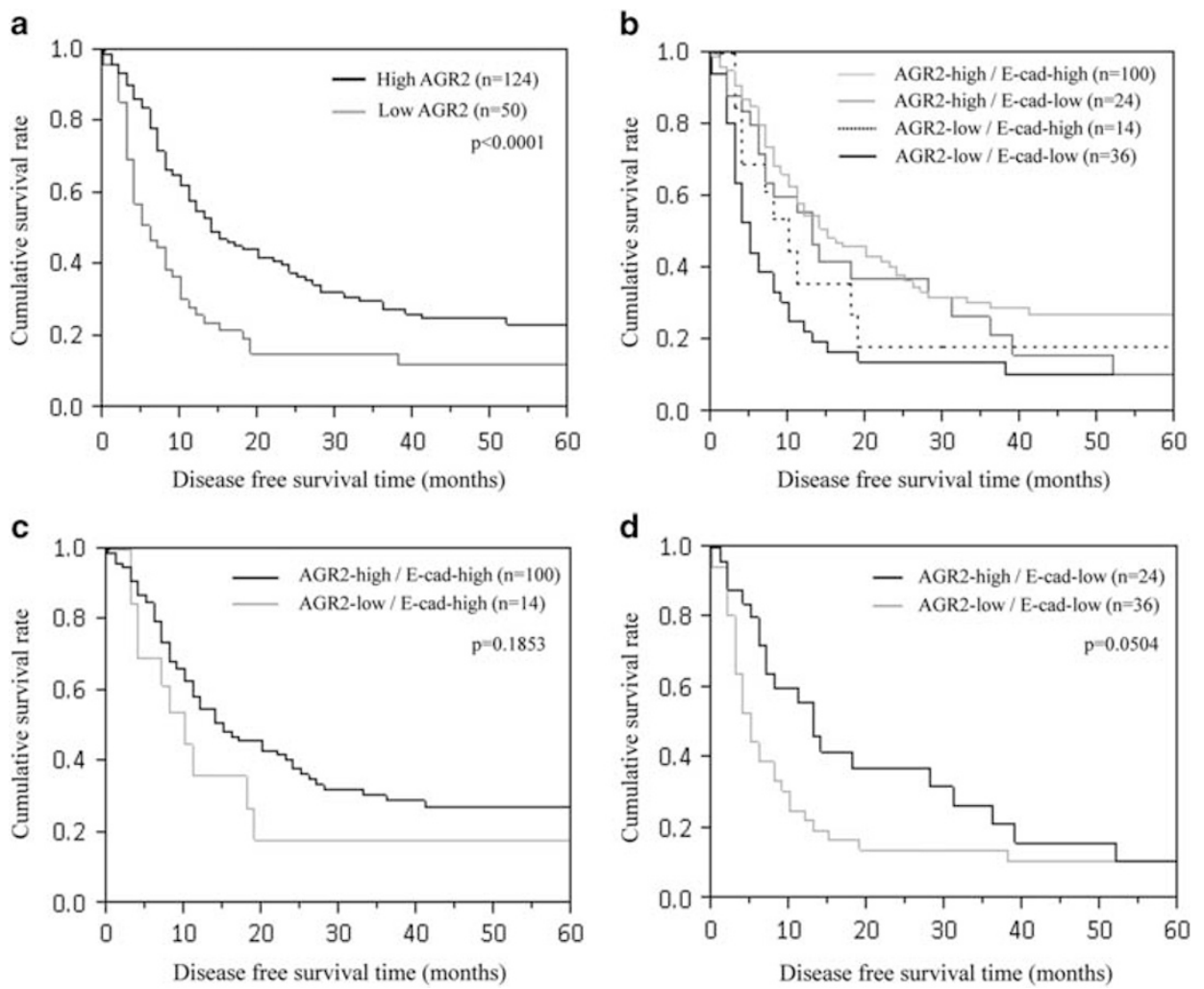

Figure 3 Kaplan-Meier survival curves demonstrating disease-free survival in PDAC patients according to anterior gradient 2 (AGR2) and E-cadherin expression. Low AGR2 expression was associated with poor prognosis in disease-free survival (a; log-rank test; $P<0.0001)$. Disease-free survival rate with combined AGR2 and E-cadherin status (b). In both high- and low-E-cadherin expression cohorts, low AGR2 expression was associated with a lower survival rate (c and $\mathbf{d}$ ).

\section{AGR2 Downregulation Mediated by TGF- $\beta$ Signaling Secreted by PSCs}

TGF- $\beta$ has previously been described as an inducer of $\mathrm{EMT}^{33}$ and a negative regulator of AGR2 ${ }^{34}$ in PDAC. We next examined total levels of secreted TGF- $\beta$ in co-culture supernatants of Aspc-1 and PSCs by ELISA. Whereas low levels of TGF- $\beta$ were secreted into the medium of Aspc-1 monocultures, the concentration of TGF- $\beta$ in co-culture supernatants was $2.5-4.5$-fold higher $(\approx 1 \mathrm{ng} / \mathrm{ml}$, Figure $6 \mathrm{e})$. AGR2 expression was decreased following treatment of Panc1, Aspc-1, and Capan-2 cells with human recombinant TGF- $\beta 1$. High levels of EGF also inhibited AGR2 expression in Aspc- 1 and Capan-2 cells, but not in Panc-1 cells, whereas treatment with FGF-2 did not alter AGR2 expression in PDAC cells (Figure 6f). In addition, the level of phosphorylated ERK1/2 was elevated in cancer cells co-cultured with PSCs compared with monocultures. Phosphorylated Smad2/3 was observed in Panc- 1 and Capan-2 cells, but not in Aspc-1 cells (Figure 6d). AGR2 downregulation was associated with phosphorylation of ERK1/2 in Panc-1, Aspc-1, and Capan-2 cells. To confirm whether AGR2 expression is reduced by the function of TGF- $\beta 1$ in PDAC cells, we performed co-culture experiments inhibiting TGF- $\beta$ signaling using a TGF- $\beta 1$ neutralizing antibody and recombinant human TGF- $\beta$ receptor type II Fc Chimera. AGR2 mRNA expression was restored in PDAC cells cocultured with PSCs following inhibition of TGF- $\beta$ signaling (Figure 6g). These results indicate that TGF- $\beta$ secreted from PSCs may partially contribute to downregulation of AGR2.

\section{DISCUSSION}

In this study, we demonstrated that AGR2 downregulation occurred in high histological grade PDAC in association with EMT induction, and correlated with poor outcome in PDAC patients. We also demonstrated that AGR2 expression was downregulated in PDAC cells following co-culture with PSCs, consistent with EMT induction, and TGF- $\beta 1$ secreted from PSCs partially contribute to its AGR2 downregulation.

Our IHC analyses revealed that AGR2 expression was upregulated during neoplastic initiation and subsequently downregulated during cancer progression, cancer cell de-differentiation, and EMT induction. This suggests that AGR2 may contribute to early neoplastic initiation by increasing proliferation and migration rather than progression and metastasis, and that downregulation of AGR2 occurs in advanced- and high-grade PDAC. IHC analyses of EMT markers in these specimens also demonstrated that AGR2 downregulation was correlated with EMT induction in 

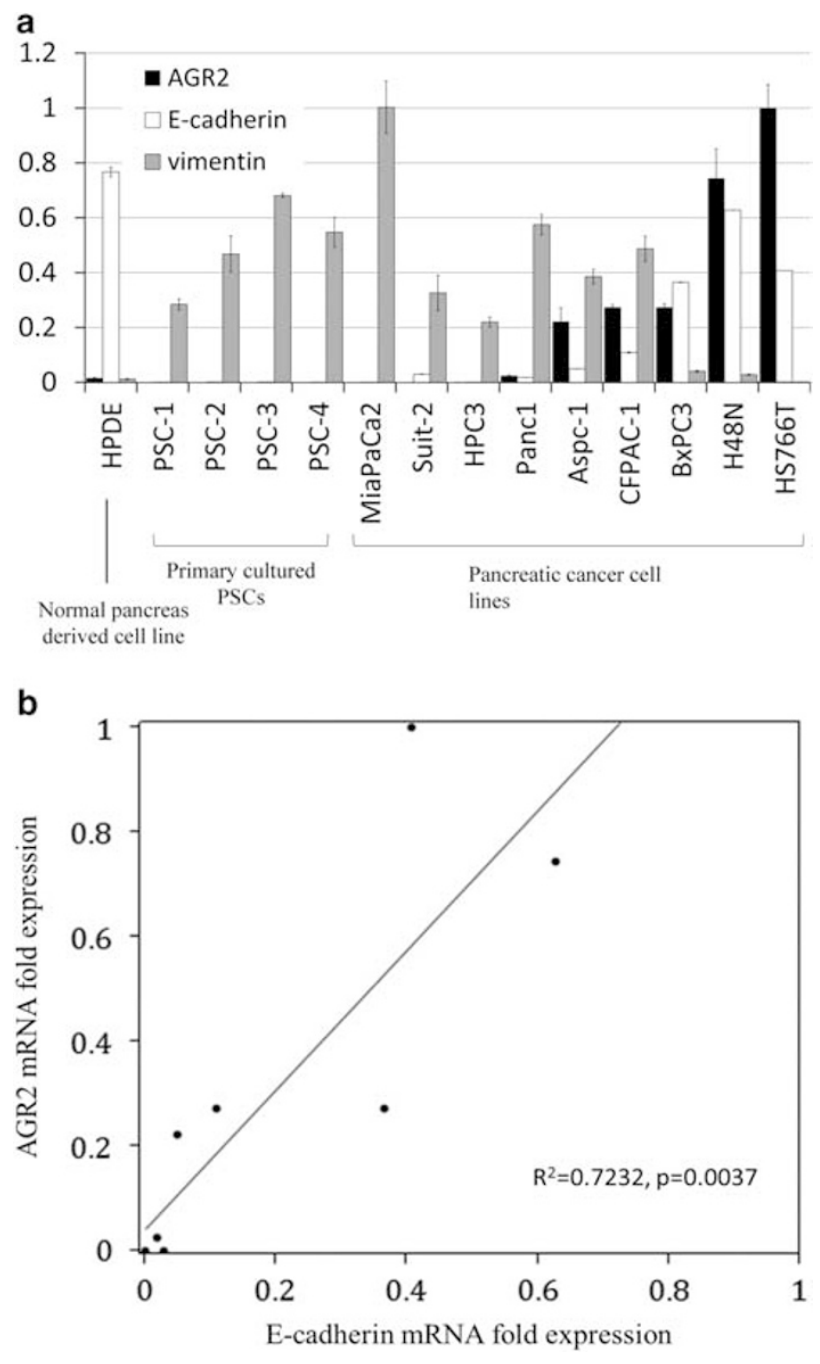

Figure 4 Quantitative reverse transcription-PCR for anterior gradient 2 (AGR2) expression in pancreatic cancer cell lines. AGR2 expression differed in the various pancreatic cancer cell lines. High AGR2-expressing cell lines exhibited overexpression of vimentin and reduced E-cadherin expression. Epithelial-type cancer cells (HS766T, H48N, and BxPC-3), retained cellular adhesiveness and AGR2 expression, whereas mesenchymal-type cancer cell lines (MiaPaCa2, SUIT-2, and HPC-3) exhibiting spindle-shaped morphology expressed low levels of AGR2. AGR2 and E-cadherin expression was very low and vimentin expression was quite high in the primary-cultured pancreatic stellate cells (PSCs) (a). Linear regression analysis demonstrated a strong positive correlation between AGR2 and E-cadherin messenger RNA (mRNA) levels (b: $\left.R^{2}=0.7262, P=0.0035\right)$.

PDAC patients. In this study, AGR2 downregulation occurred in only $28 \%$ of PDAC patients. It should be noted that AGR2 expression can also be upregulated in PDAC, as other publications have suggested. ${ }^{14,16}$

Previous reports have documented conflicting results regarding the prognostic value of AGR2 expression. In prostate cancer tissue, AGR2 downregulation correlated with increased Gleason grade, a histological grading system of prostate cancer, and increased cancer recurrence in Gleason grades 4 or $5 .{ }^{35} \mathrm{~A}$ more recent report demonstrated that high
AGR2 expression and low CD10 expression were favorable prognostic markers in prostate cancer. ${ }^{36}$ In contrast, another reported that increased AGR2 expression was correlated with poorer outcome in prostate cancer. ${ }^{13}$ Similar controversy regarding the prognostic value of AGR2 expression has been observed in breast cancer; with AGR2 upregulation associated with favorable prognosis in one study, ${ }^{37}$ and with poor prognosis in another independent analysis. ${ }^{11}$

Previous studies of PDAC did not identify a correlation between AGR2 expression and prognosis. ${ }^{15}$ Our univariate analysis revealed that AGR2 downregulation correlated with poor prognosis in PDAC patients. In contrast, multivariate analysis revealed that AGR2 expression was not a poor prognostic marker, whereas downregulation of E-cadherin had significant prognostic value (Supplementary Table 2). Furthermore, division of patients into two groups based on E-cadherin expression reveals that low-AGR2 expression tends to be an indicator of shorter survival, although this difference was not statistically significant (Figures $3 \mathrm{c}$ and d). It can be said that AGR2 has a strong correlation with E-cadherin and makes it as a strong confounding factor. Indeed, exclusion of E-cadherin expression from the multivariate analysis revealed that AGR2 downregulation was an independent prognostic marker (data not shown), suggesting the strong correlation between AGR2 downregulation and decreased membranous E-cadherin expression. In the view of a prognostic marker, as AGR2 is secreted into the pancreatic fluid, unlike E-cadherin or vimentin, ${ }^{38}$ it may represent a useful and convenient pre-operative prognostic marker. To the best of our knowledge, our study is the first report demonstrating the prognostic significance of decreased AGR2 expression in PDAC patients.

Whereas AGR2 has been previously described as an oncoprotein capable of promoting cancer cell proliferation, invasiveness, and migration in various PDAC cell lines, ${ }^{14}$ our IHC study revealed that AGR2 was downregulated during PDAC progression and this was associated with poor prognosis. Consistent with our IHC results in PDAC, recent reports reveal that AGR2 immunoreactivity is frequently lost in colorectal carcinoma, and may represent a novel prognostic indicator for overall patient survival. ${ }^{39}$

Discrepancy between pro-oncogenic function of AGR2 as previously reported and the prognostic significance of AGR2 downregulation may have several explanations. First, the biological outcome of altered AGR2 expression may depend on the origin of tumor development. For example, caveolin1 , an essential constituent protein of specialized membrane invaginations referred to as caveolae, was upregulated in prostate cancer tissue ${ }^{40}$ but downregulated in colon cancer tissue. $^{41}$ Targeted knockdown of AGR2 in PDAC cells using siRNA revealed that AGR2 silencing suppressed the proliferation, invasiveness, and migration of Aspc-1 and Hs766T cells, as previously reported in other PDAC cell lines (BxPC-3, SU8686, CFPAC-1, and MPanc-96), ${ }^{14}$ but did not alter EMT markers. These data suggest that AGR2 promotes cancer 

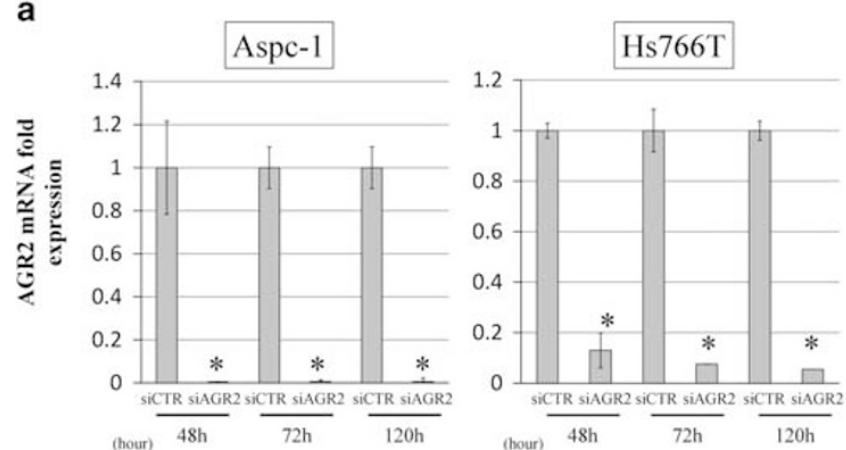

C

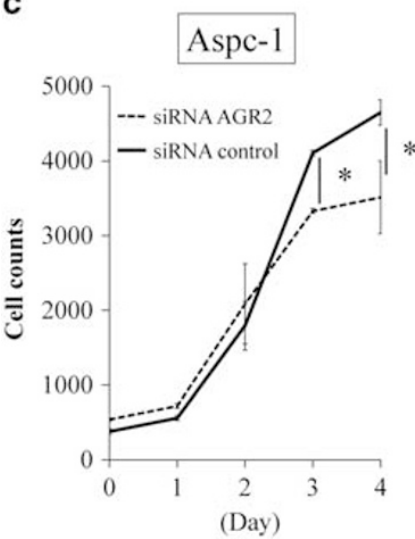

e

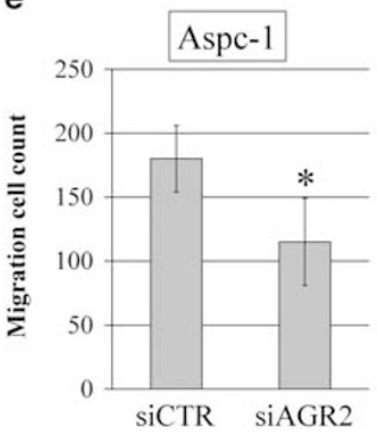

\section{Hs766T}
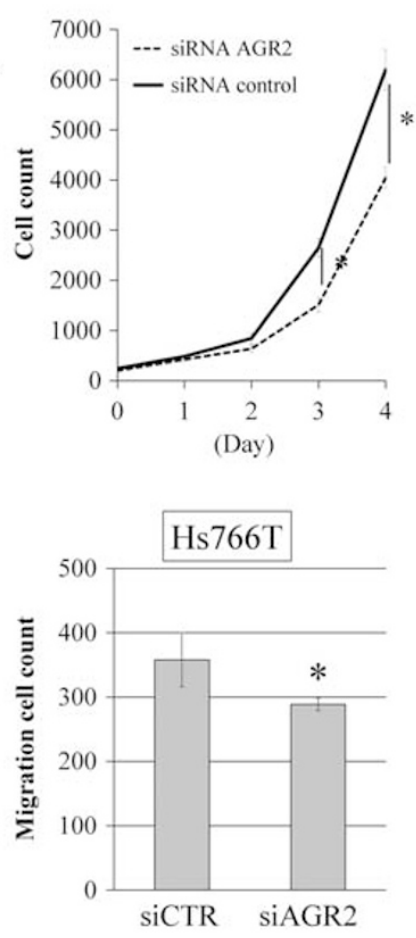

b

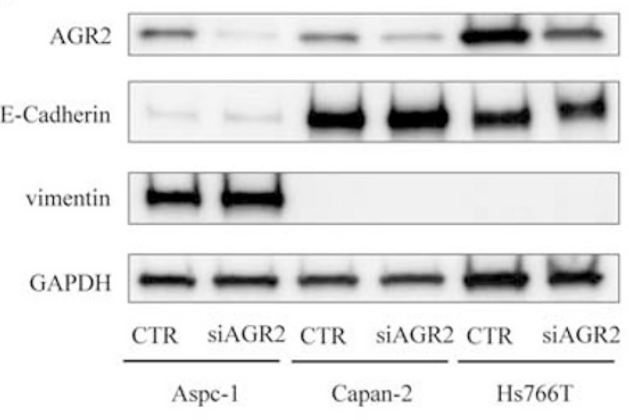

d

Hs766T
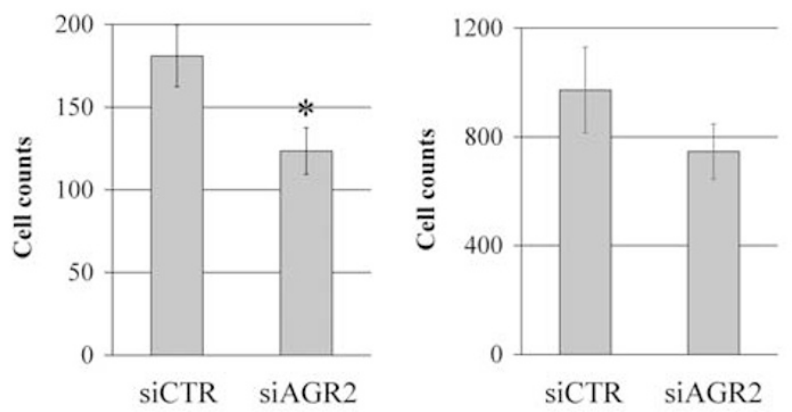

f

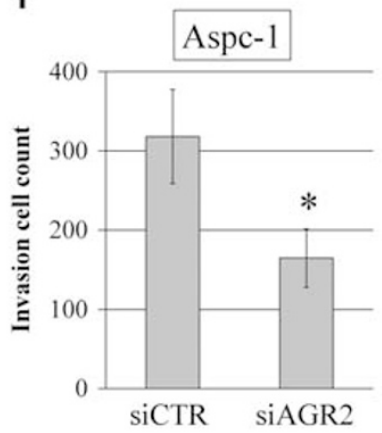

g

9

-AGR2 $\square$ E-cadherin $\square$ Vimentin $\square$ Snail-1 $\square$ Snail-2 $\backsim$ ZEB-1

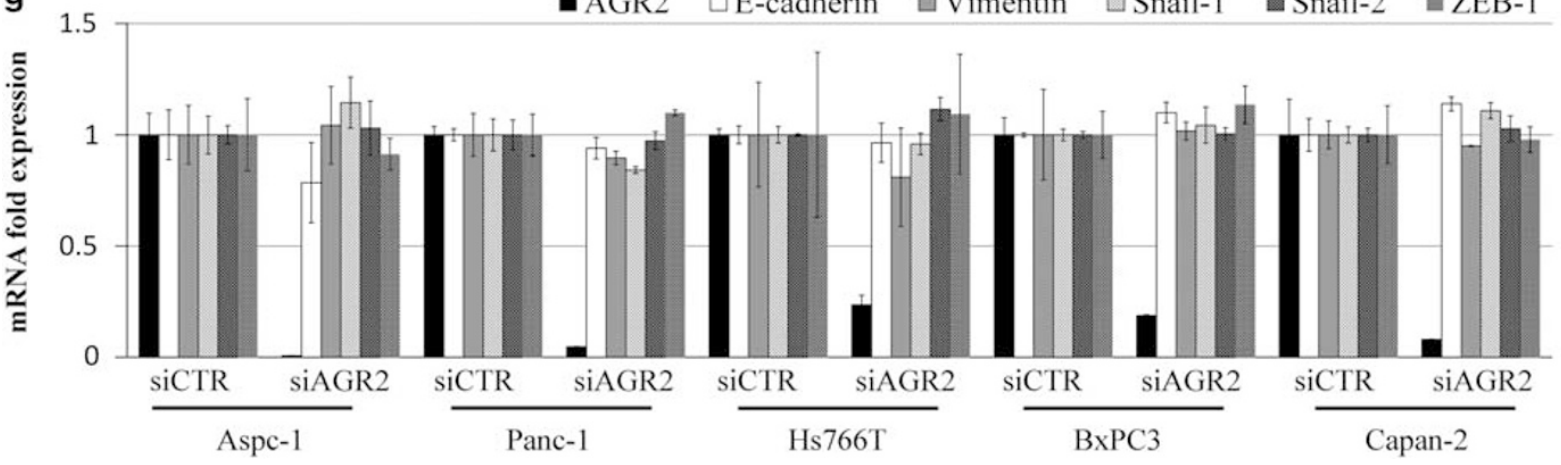

Figure 5 Targeted inhibition of anterior gradient 2 (AGR2) using small-interfering RNA (siRNA). AGR2 silencing was confirmed following transfection of Aspc-1 and Hs766T cells with AGR2-specific siRNA by quantitative reverse transcription (qRT)-PCR (a) 48, 72, and 120 h, and immunoblot analysis 72 h post transfection, respectively (b). E-cadherin and vimentin expression levels were not altered by AGR2 knockdown as assessed by immunoblot analysis (b). AGR2 knockdown significantly reduced cell proliferation (c), colony formation (d), cell migration (e), and cell invasiveness (f) in Aspc-1 and Hs766T cells $(P<0.01)$, except for the colony formation assay in Hs766T cells $(P=0.0686)$. AGR2 knockdown did not alter messenger RNA (mRNA) levels of epithelial-mesenchymal transition markers, E-cadherin, vimentin, Snail-1, Snail-2, and ZEB-1 (g). Data represent the mean values of triplicate experiments ( ${ }^{*} P<0.01$, versus control). GAPDH, glyceraldehyde 3-phosphate dehydrogenase. 
a
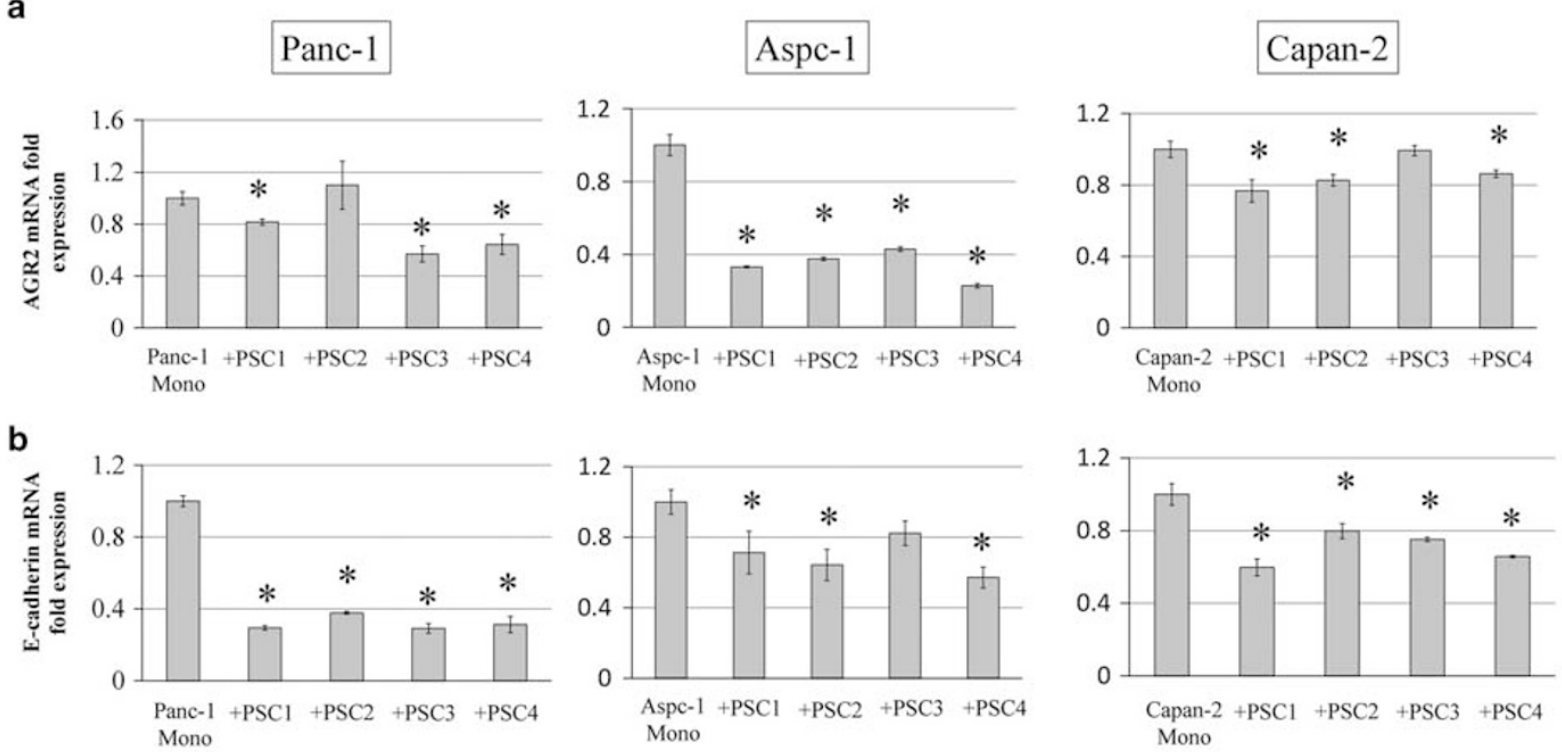

C
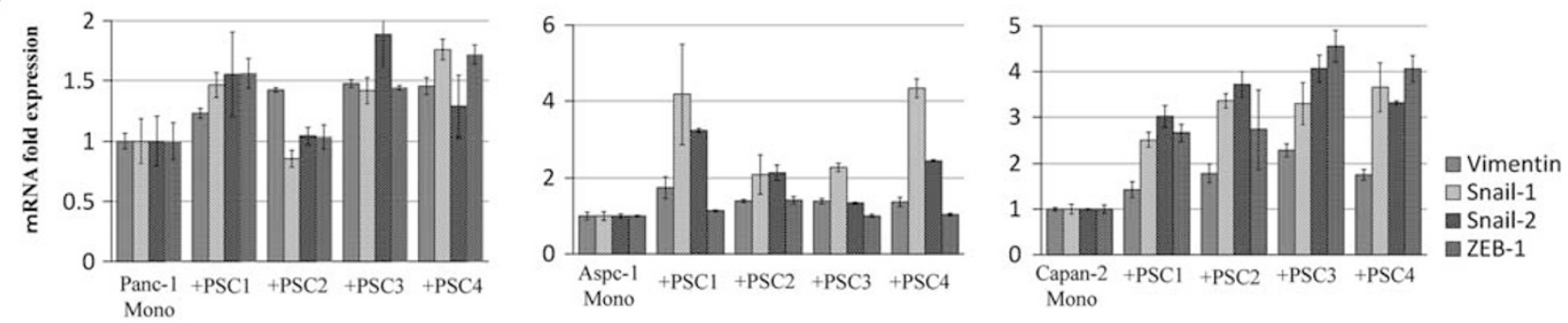

d

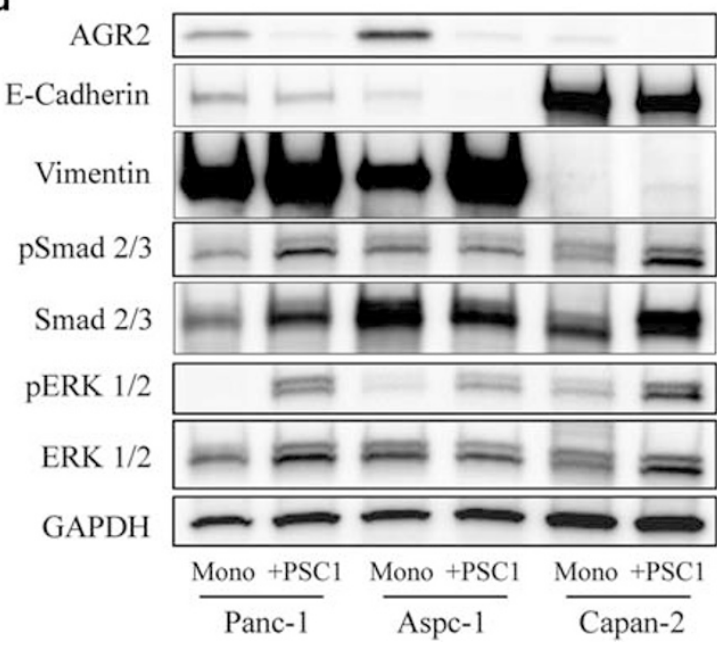

e

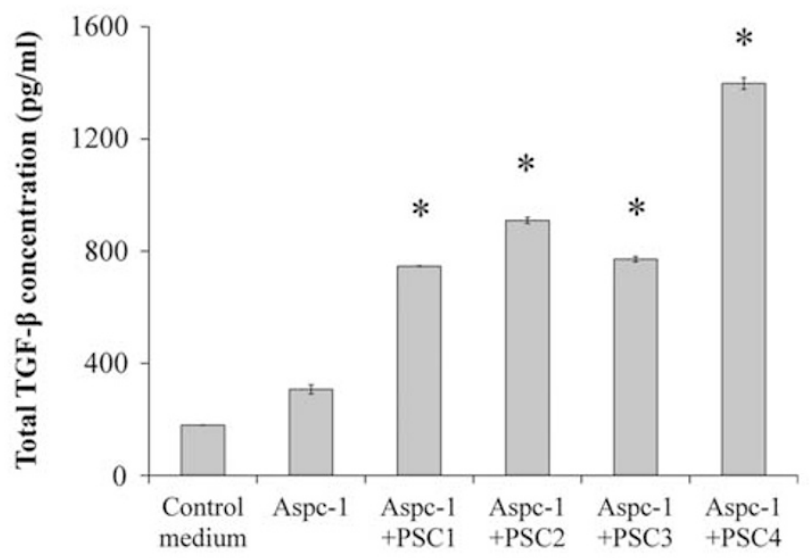

Figure 6 Indirect co-culture experiment of pancreatic ductal adenocarcinoma (PDAC) cells with pancreatic stellate cell (PSCs) and treatment with recombinant growth factors. Anterior gradient 2 (AGR2) expression was decreased in PDAC cells co-cultured with PSCs using an indirect co-culture system (a) in accordance with epithelial-mesenchymal transition induction (E-cadherin downregulation (b) and overexpression of vimentin, Snail-1, Snail-2 and ZEB-1 (c)) by quantitative reverse transcription-PCR ( ${ }^{*} P<0.01$, versus monocultures). Decreased expression of AGR2 was observed in cocultured cancer cells compared with monocultures. Decreased AGR2 expression was also associated with increased levels of phosphorylated Smad2/3 and ERK1/2, with the exception of phosphorylated Smad2/3 in Aspc-1 (d). E-cadherin and vimentin expression were also slightly altered at the protein level (d). Enzyme-linked immunosorbent assays revealed that total, secreted transforming growth factor beta-1 (TGF- $\beta 1$ ) levels were higher in co-culture supernatants than that of monocultures (e: ${ }^{*} P<0.001$, versus the medium of Aspc-1 monocultures). Treatment with recombinant proteins showed that reduced expression of AGR2 was observed by TGF- $\beta 1$ in all PDAC cells examined and by high-dose epidermal growth factor (EGF) in Aspc-1 and Capan-2 cells (f). AGR2 expression was decreased by TGF- $\beta 1$ or co-cultured with PSCs, and restored by inhibition of TGF- $\beta$ signaling (g). Data represent the mean values of triplicate experiments ( ${ }^{*} P<0.01$, versus control). GAPDH, glyceraldehyde 3-phosphate dehydrogenase; FGF-2, fibroblast growth factor 2; mRNA, messenger RNA. 
f
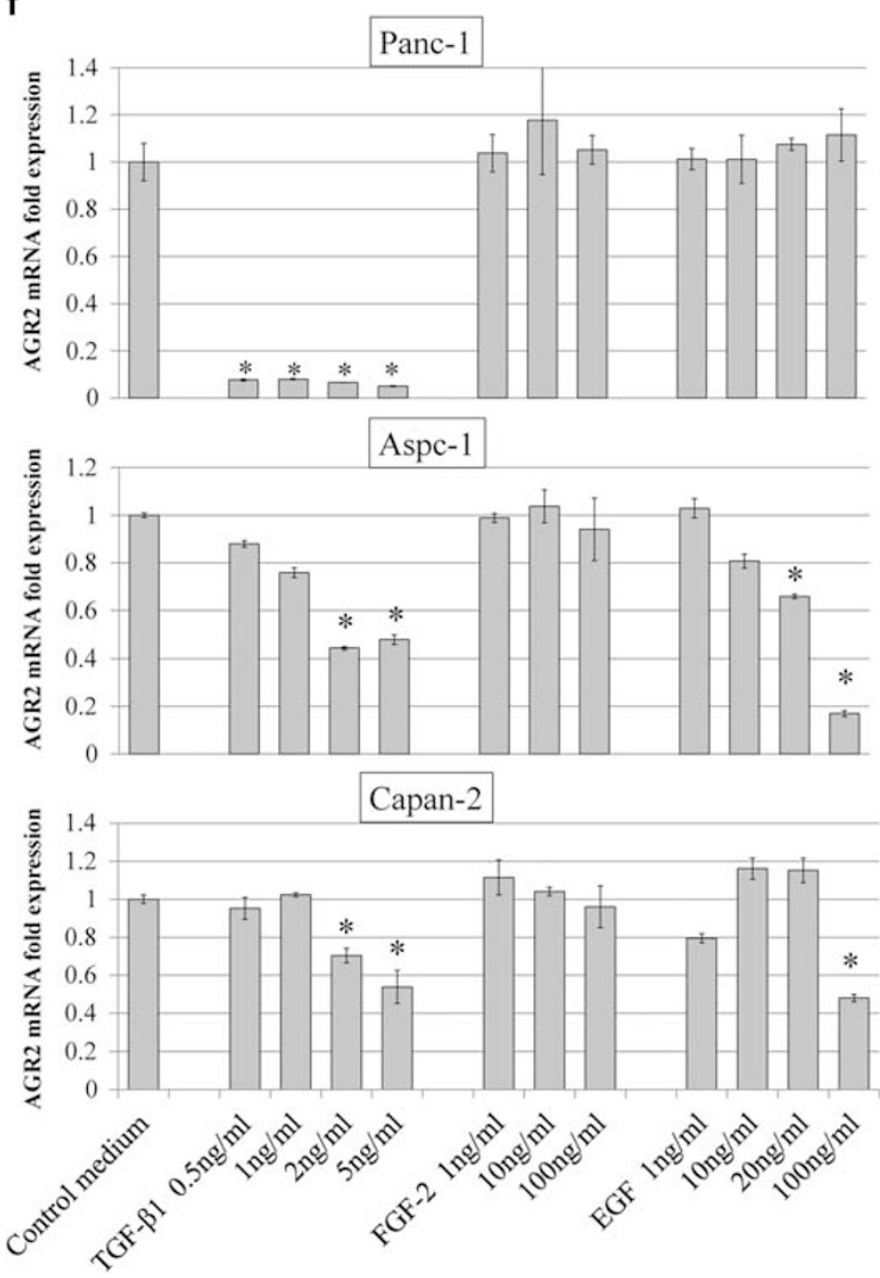

g

Panc-1

Aspc-1

Capan-2
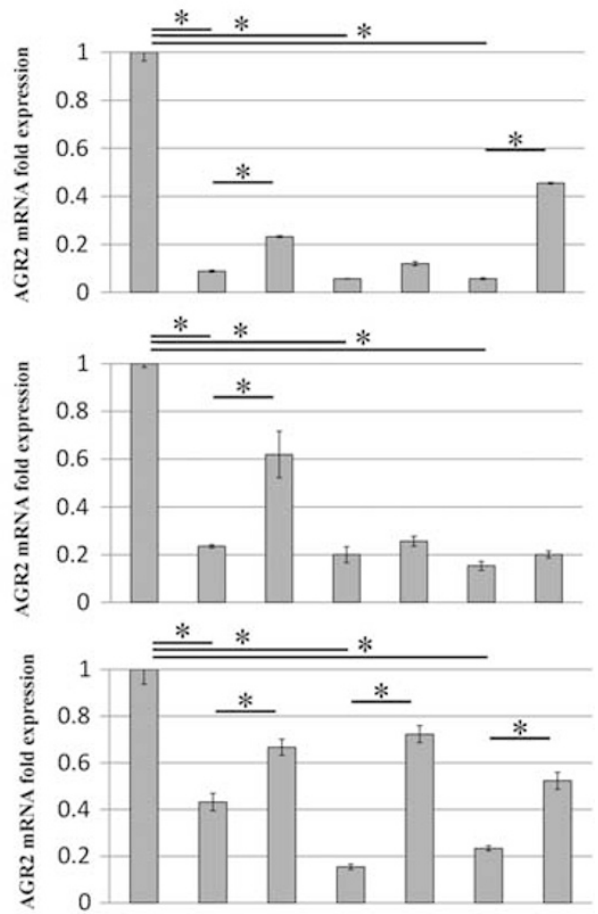

TGF- $\beta 11 \mathrm{ng} / \mathrm{ml}$

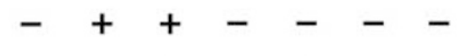

rhTßsR-II 100ng/ml $-\quad+-+-$

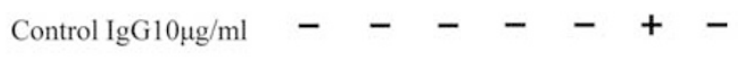

TGF- $\beta$ Ab $10 \mu \mathrm{g} / \mathrm{ml}-\infty \quad-\quad-\quad+$

Cocultured with PSC-1 $-\quad-\quad+++$

Figure 6 Continued.

progression, but does not induce EMT or mesenchymalepithelial transition in PDAC patients. Second, the effect of AGR2 expression may also be dependent on tumor stage. IHC analyses revealed that AGR2 was expressed in precursor PDAC lesions, and its expression was higher than in PDAC. It was proposed that the pro-oncogenic function of AGR2 may be active during early tumor development and promote neoplastic initiation, with TGF- $\beta$ secreted from PSCs in the cancer stroma functioning as an upstream negative regulator of AGR2 at the late stage of cancer progression. Thus, it was suggested that the oncogenic function of AGR2 exerted little influence on prognosis in PDAC patients. Third, AGR2 downregulation in PDAC was caused by morphological and phenotypic alterations during pancreatic cancer progression. We demonstrated that AGR2 expression was decreased in parallel with EMT induction, characterized by decreased membranous E-cadherin expression and increased cytoplasmic vimentin expression in IHC analyses. On the basis of these observations, we hypothesized that AGR2 downregulation may be mediated by upstream regulators that function as EMT inducers.
PSCs are one of the most important cell populations in the pancreatic cancer stroma. PSCs are also observed surrounding PanIN lesions; however, it has been reported that the cellular density of PSCs is lower than that of PDAC, and that the phenotypic characteristics of PSCs surrounding PanINs may be different from that in PDAC. ${ }^{42}$ As cancer cells invade the stroma, destructing the basement membrane, they interact with stromal cells including PSCs. This interaction promotes tumor formation and metastasis induced by secreted soluble factors from stromal cells, including various growth factors and cytokines. Whereas AGR2 itself promotes cancer initiation in PDAC development, it was suggested that other prooncogenic factors secreted from cancer microenvironments also contribute to cancer cell invasiveness. AGR2 expression was consequently reduced following induction of EMT (alteration to mesenchymal morphology) and the acquisition of a highly aggressive phenotype by these secreted factors. To identify such soluble factors associated with AGR2 downregulation in PDAC cells, we cultured PDAC cells with culture medium containing various recombinant growth factors known as EMT-inducible factors and secreted from 


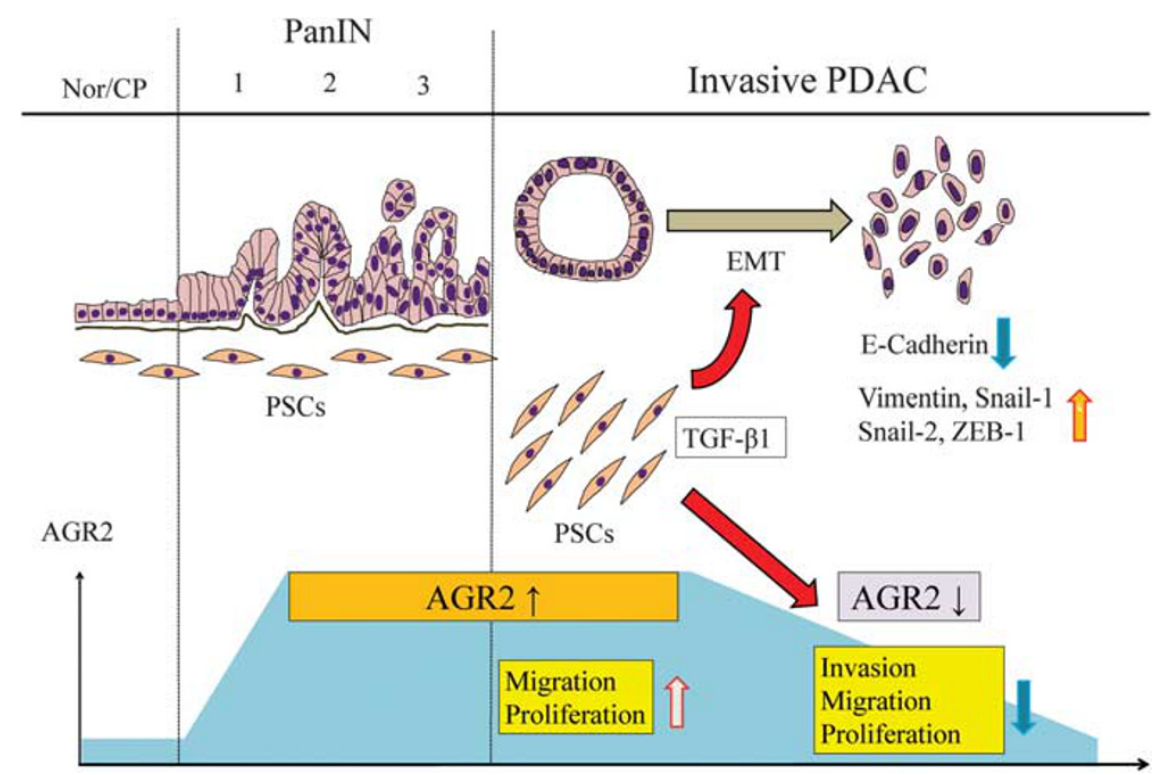

Figure 7 Schematic summarising the mechanisms of anterior gradient 2 (AGR2) downregulation. AGR2 expression was not observed in non-neoplastic epithelium of normal pancreas or chronic pancreatitis, but was upregulated in intraepithelial neoplastic lesions and contributed to cancer development. As cancer cells invade the stroma through the basement membrane, EMT is induced following interaction with stromal cells, including PSCs. AGR2 is downregulated by TGF- $\beta 1$ secreted from PSCs. Cancer progression is slightly reduced by AGR2 downregulation; however, the various oncogenic factors, upregulated in advanced PDAC, retain or promote cancer progression. (Nor/CP, pancreatic ductal epithelium of normal pancreas and chronic pancreatitis; PanIN, pancreatic intraepithelial epithelium; PDAC, pancreatic ductal adenocarcinoma; EMT, epithelial-mesenchymal transition; PSCs, pancreatic stellate cells; TGF- $\beta 1$, transforming growth factor beta-1).

PSCs, suggesting that AGR2 downregulation may occur in advanced PDAC cells by this factor secreted from PSCs in cancer stroma.

A recent study demonstrated that AGR2 expression is suppressed in Panc-1 cells with wild-type Smad4 by a canonical TGF- $\beta /$ Smad signaling pathway; ${ }^{34}$ however, our study revealed that TGF- $\beta$ treatment reduced AGR2 expression in not only Capan-2 and Panc-1, but also Aspc-1 cells harboring Smad4 mutation. ${ }^{43}$ The concentration of TGF- $\beta$ in cell supernatants co-cultured with PSCs, as assessed by ELISA, was $\approx 1000 \mathrm{pg} / \mathrm{ml}$. Importantly, this level was sufficient to mediate AGR2 downregulation. TGF- $\beta$ also stimulates phosphorylation of ERK1/2 through a Smad-independent pathway. ${ }^{44}$ Co-culture experiments demonstrated that phosphorylation of ERK1/2 occurred in parallel with AGR2 downregulation. This suggests that TGF- $\beta$ signaling was activated in the PDAC co-culture setting regardless of DPC4 mutation, and TGF- $\beta$-mediated downregulation of AGR2 also occurs via a Smad-independent pathway, although the exact mechanism(s) underlying the downregulation of AGR2 by TGF- $\beta$ signaling remains unclear. These results are schematically summarized in Figure 7.

Collectively, although the complete mechanisms of AGR2 downregulation accompanying EMT induction remain unclear, we demonstrated that AGR2 is downregulated in PDAC compared with pre-malignant lesions, and AGR2 expression may be a favorable prognostic marker. Targeted silencing of
AGR2 using siRNA revealed that AGR2 promotes cancer cell proliferation, migration, and invasion, confirming previous reports that AGR2 itself functions as an oncoprotein. Reduced AGR2 expression was detected in PDAC cell lines co-cultured with primary-cultured PSC, in parallel with EMT induction. These results suggest that reduced AGR2 expression does not cause EMT, but is the result of aggressive phenotype in advanced PDAC. Supplementary Information accompanies the paper on the Laboratory
Investigation website (http://www.laboratoryinvestigation.org)

\section{ACKNOWLEDGMENTS}

We thank N Tateishi (Department of Anatomic Pathology, Kyushu University Hospital) and E Manabe (Department of Surgery and Oncology, Kyushu University Hospital) for their expert technical assistance. This study was supported by a Grant-in-Aid for the Japan Society for the Promotion of Science Fellows (13-3401). We also thank Edanz Group Japan for revising the English used in this article.

\section{DISCLOSURE/CONFLICT OF INTEREST}

The authors declare no conflict of interest.

1. Siegel R, Naishadham D, Jemal A. Cancer statistics, 2012. CA Cancer J Clin 2012;62:10-29.

2. Hruban RH, Wilentz RE, Kern SE. Genetic progression in the pancreatic ducts. Am J Pathol 2000;156:1821-1825. 
3. van Heek T, Rader AE, Offerhaus GJ, et al. K-ras, p53, and DPC4 (MAD4) alterations in fine-needle aspirates of the pancreas: a molecular pane correlates with and supplements cytologic diagnosis. Am J Clin Pathol 2002;117:755-765.

4. Hansel DE, Kern SE, Hruban RH. Molecular pathogenesis of pancreatic cancer. Annu Rev Genomics Hum Genet 2003;4:237-256.

5. Sive $\mathrm{HL}$, Hattori $\mathrm{K}$, Weintraub $\mathrm{H}$. Progressive determination during formation of the anteroposterior axis in Xenopus laevis. Cell 1989; 58:171-180.

6. Thompson DA, Weigel RJ. HAG-2, the human homologue of the Xenopus laevis cement gland gene XAG-2, is coexpressed with estrogen receptor in breast cancer cell lines. Biochem Biophys Res Commun 1998;251:111-116.

7. Aberger $\mathrm{F}$, Weidinger $\mathrm{G}, \mathrm{Grunz} \mathrm{H}$, et al. Anterior specification of embryonic ectoderm: the role of the Xenopus cement gland-specific gene XAG-2. Mech Dev 1998;72:115-130.

8. Kumar A, Godwin JW, Gates PB, et al. Molecular basis for the nerve dependence of limb regeneration in an adult vertebrate. Science 2007;318:772-777.

9. Brychtova V, Vojtesek B, Hrstka R. Anterior gradient 2: a novel player in tumor cell biology. Cancer Lett 2011;304:1-7.

10. Darb-Esfahani S, Fritzsche F, Kristiansen G, et al. Anterior gradient protein 2 (AGR2) is an independent prognostic factor in ovarian highgrade serous carcinoma. Virchows Arch 2012;461:109-116.

11. Innes HE, Liu D, Barraclough $R$, et al. Significance of the metastasisinducing protein AGR2 for outcome in hormonally treated breast cancer patients. Br J Cancer 2006:94:1057-1065.

12. Wang Z, Hao Y, Lowe AW. The adenocarcinoma-associated antigen, AGR2, promotes tumor growth, cell migration, and cellular transformation. Cancer Res 2008;68:492-497.

13. Zhang Y, Forootan SS, Liu D, et al. Increased expression of anterior gradient-2 is significantly associated with poor survival of prostate cancer patients. Prostate Cancer Prostatic Dis 2007:10:293-300.

14. Ramachandran V, Arumugam T, Wang $\mathrm{H}$, et al. Anterior gradient 2 is expressed and secreted during the development of pancreatic cancer and promotes cancer cell survival. Cancer Res 2008;68:7811-7818.

15. Riener MO, Pilarsky C, Gerhardt J, et al. Prognostic significance of AGR2 in pancreatic ductal adenocarcinoma. Histol Histopathol 2009;24: 1121-1128.

16. Dumartin L, Whiteman HJ, Weeks ME, et al. AGR2 is a novel surface antigen that promotes the dissemination of pancreatic cancer cells through regulation of cathepsins B and D. Cancer Res 2011;71:70917102.

17. Hugo $\mathrm{H}$, Ackland $\mathrm{ML}$, Blick $\mathrm{T}$, et al. Epithelial-mesenchymal and mesenchymal-epithelial transitions in carcinoma progression. J cell Physiol 2007:213:374-383.

18. Prudkin L, Liu DD, Ozburn NC, et al. Epithelial-to-mesenchymal transition in the development and progression of adenocarcinoma and squamous cell carcinoma of the lung. Mod Pathol 2009;22: 668-678.

19. Morali OG, Delmas V, Moore R, et al. IGF-II induces rapid beta-catenin relocation to the nucleus during epithelium to mesenchyme transition. Oncogene 2001;20:4942-4950.

20. Jeong $\mathrm{H}$, Ryu YJ, An J, et al. Epithelial-mesenchymal transition in breast cancer correlates with high histological grade and triplenegative phenotype. Histopathology 2012;60:E87-E95

21. Bachem MG, Schneider $\mathrm{E}$, Gross $\mathrm{H}$, et al. Identification, culture, and characterization of pancreatic stellate cells in rats and humans. Gastroenterology 1998;115:421-432.

22. Erkan M, Adler G, Apte MV, et al. StellaTUM: current consensus and discussion on pancreatic stellate cell research. Gut 2012;61:172-178.

23. Hwang RF, Moore T, Arumugam T, et al. Cancer-associated stromal fibroblasts promote pancreatic tumor progression. Cancer Res 2008;68:918-926.
24. Miettinen PJ, Ebner $\mathrm{R}$, Lopez $\mathrm{AR}$, et al. TGF-beta induced transdifferentiation of mammary epithelial cells to mesenchymal cells: involvement of type I receptors. J Cell Biol 1994;127:2021-2036.

25. Sakuma K, Aoki M, Kannagi R. Transcription factors C-Myc and CDX2 mediate E-selectin ligand expression in colon cancer cells undergoing EGF/bFGF-induced epithelial-mesenchymal transition. Proc Natl Acad Sci USA 2012;109:7776-7781.

26. El-Hariry I, Pignatelli M, Lemoine NR. FGF-1 and FGF-2 regulate the expression of $\mathrm{E}$-cadherin and catenins in pancreatic adenocarcinoma. Int J Cancer 2001;94:652-661.

27. Kikuta K, Masamune A, Watanabe T, et al. Pancreatic stellate cells promote epithelial-mesenchymal transition in pancreatic cancer cells. Biochem Biophys Res Commun 2010;403:380-384.

28. Al-Aynati MM, Radulovich N, Riddell RH, et al. Epithelial-cadherin and b-catenin expression changes in pancreatic intraepithelial neoplasia. Clin Cancer Res 2004;10:1235-1240.

29. Ohuchida K, Mizumoto K, Ishikawa N, et al. The role of S100A6 in pancreatic cancer development and its clinical implication as a diagnostic marker and therapeutic target. Clin Cancer Res 2005;11: 7785-7793.

30. Ikenaga N, Ohuchida K, Mizumoto K, et al. CD10 + pancreatic stellate cells enhance the progression of pancreatic cancer. Gastroenterology 2010;139:1041-1051.

31. Odate $\mathrm{S}$, Nakamura $\mathrm{K}$, Onishi $\mathrm{H}$, et al. TrkB/BDNF signaling pathway is a potential therapeutic target for pulmonary large cell neuroendocrine carcinoma. Lung Cancer 2013;79:205-214.

32. Tsang ML, Zhou $\mathrm{L}$, Zheng BL, et al. Characterization of recombinant soluble human transforming growth factor-beta receptor type II (rhTGF-beta sRII). Cytokine 1995;7:389-397.

33. Ellenrieder V, Hendler SF, Boeck W, et al. Transforming growth factor beta1 treatment leads to an epithelial-mesenchymal transdifferentiation of pancreatic cancer cells requiring extracellular signalregulated kinase 2 activation. Cancer Res 2001;61:4222-4228.

34. Norris AM, Gore A, Balboni A, et al. AGR2 is a SMAD4-suppressible gene that modulates MUC1 levels and promotes the initiation and progression of pancreatic intraepithelial neoplasia. Oncogene 2013;32:3867-3876.

35. Maresh EL, Mah V, Alavi M, et al. Differential expression of anterior gradient gene AGR2 in prostate cancer. BMC Cancer 2010;10:680.

36. Ho ME, Quek SI, True LD, et al. Prostate cancer cell phenotypes based on AGR2 and CD10 expression. Mod Pathol 2013;26:849-859.

37. Fritzsche FR, Dahl $\mathrm{E}$, Pahl $\mathrm{S}$, et al. Prognostic relevance of AGR2 expression in breast cancer. Clin Cancer Res 2006;12:1728-1734.

38. Chen R, Pan S, Duan X, et al. Elevated level of anterior gradient- 2 in pancreatic juice from patients with pre-malignant pancreatic neoplasia. Mol Cancer 2010;9:149.

39. Riener MO, Thiesler T, Hellerbrand C, et al. Loss of Anterior gradient-2 expression is an independent prognostic factor in colorectal carcinomas. Eur J Cancer 2014;50:1722-1730.

40. Yang G, Truong LD, Wheeler TM, et al. Caveolin-1 expression in clinically confined human prostate cancer: a novel prognostic marker. Cancer Res 1999;59:5719-5723.

41. Bender FC, Reymond MA, Bron C, et al. Caveolin-1 levels are downregulated in human colon tumors, and ectopic expression of caveolin1 in colon carcinoma cell lines reduces cell tumorigenicity. Cancer Res 2000;60:5870-5878.

42. Shi C, Washington MK, Chaturvedi R, et al. Fibrogenesis in pancreatic cancer is a dynamic process regulated by macrophage-stellate cell interaction. Lab Invest 2014;94:409-421.

43. Schutte $M$, Hruban RH, Hedrick $L$, et al. DPC4 gene in various tumor types. Cancer Res 1996;56:2527-2530.

44. Zavadil J, Bitzer M, Liang D, et al. Genetic programs of epithelial cell plasticity directed by transforming growth factor-beta. Proc Natl Acad Sci USA 2001;98:6686-6691. 